\title{
Thrust generation and wake structure of wiggling hydrofoil*
}

\author{
Guo-yi HE (何国毅) $)^{1,2}$, Shu-guang ZHANG (张曙光) ${ }^{3}, \quad$ Xing ZHANG (张 星) ${ }^{4}$ \\ (1. School of Aircraft Engineering, Nanchang Hangkong University, \\ Nanchang 330063, P. R. China; \\ 2. School of Aeronautic Science and Engineering, Beihang University, \\ Beijing 100191, P. R. China; \\ 3. School of Transportation Science and Engineering, Beihang University, \\ Beijing 100191, P. R. China; \\ 4. The State key Laboratory of Nonlinear Mechanics, Institute of Mechanics, \\ Chinese Academy of Sciences, Beijing 100080, P. R. China) \\ (Communicated by Ji-sheng LUO)
}

\begin{abstract}
Marine animals and micro-machines often use wiggling motion to generate thrust. The wiggling motion can be modeled by a progressive wave where its wavelength describes the flexibility of wiggling animals. In the present study, an immersed boundary method is used to simulate the flows around the wiggling hydrofoil NACA 65-010 at low Reynolds numbers. One can find from the numerical simulations that the thrust generation is largely determined by the wavelength. The thrust coefficients decrease with the increasing wavelength while the propulsive efficiency reaches a maximum at a certain wavelength due to the viscous effects. The thrust generation is associated with two different flow patterns in the wake: the well-known reversed Karman vortex streets and the vortex dipoles. Both are jet-type flows where the thrust coefficients associated with the reversed Karman vortex streets are larger than the ones associated with the vortex diploes.
\end{abstract}

Key words propulsive performance, wiggling motion, immersed boundary method, wake

Chinese Library Classification O351

2000 Mathematics Subject Classification 76D17, 76Z10

\section{Introduction}

Micro-machines are broadly applied to drug delivery and surface treatment in bio-medical engineering. One strategy to propel such micro-machines is to mimic the thrust-generation mechanism of a swimming eel. Eels generate thrust by wiggling their bodies into a backward moving wave that extends from their heads to the tails. Lighthill used flexible hydrofoils to model the eels and expressed the wiggling motion by the progressive waves ${ }^{[1]}$, where the

* Received Jun. 17, 2009 / Revised Apr. 6, 2010

Corresponding author Xing ZHANG, Associate Professor, E-mail: zhangx@lnm.imech.ac.cn 
wavelength and amplitude represent the flexibility and magnitude of the wiggling motion, respectively. This type of motion is found to be more efficient in the thrust generation if compared with jet propulsion, screw propeller, and flagellar motion, etc. Therefore, much effort has been put into the design and construction of micro-machines propelled by the wiggling motion. Understanding the effect of the wavelength on the thrust generation is of vital importance in the design of such machines.

The thrust generation is closely related to the wake structures behind wiggling hydrofoils. Lighthill found that the wiggling motion could generate the reserved Karman vortex streets and thus produced thrust ${ }^{[2]}$. The finding is supported by the experiments on oscillating foils ${ }^{[3-5]}$. However, such theoretical analyses made by Lighthill ${ }^{[1,2,6]}, \mathrm{Wu}^{[7]}$, Newman ${ }^{[8]}$, and Cheng et al. ${ }^{[9]}$ are limited to inviscid flows. In some recent experimental and numerical studies $^{[10-16]}$, the propulsive efficiency is found to be related to the wiggling frequency and wavelength, and the vortex structure is related to the propulsive performance. Uchiyama et al. ${ }^{[17]}$ simulated the flows passing the wiggling hydrofoils without addressing the vortex structures.

In this study, the immersed boundary method ${ }^{[18-21]}$ is used to investigate the effects of wiggling wavelengths on the thrust generation. The wiggling body is modeled by the hydrofoil NACA 65-010. The thrust, lift, power, and efficiency are calculated. Two different wake structures (the Karman vortex streets and the vortex dipoles) that are obtained from the numerical simulations and their relation with thrust generation will be analyzed. The rest of this paper is organized as follows. Section 2 presents the formulation of the problem and describes the governing equations with boundary conditions. The numerical method used in this study is also given in detail. Section 3 presents the numerical results on the thrust, lift, and efficiency under different wavelengths and Reynolds numbers. The wake structures are also discussed with the relation of the thrust generation. The conclusions are presented in Section 4 .

\section{Mathematical model and numerical method}

\subsection{Wiggling hydrofoil}

We use the hydrofoil NACA 65-010, which is a typical low-speed hydrofoil, to investigate the thrust generation of wiggling micro-machines. A schematic representation of the configuration is shown in Fig. 1, where the chord length of the hydrofoil is $L$. A coordinate system is fixed at the head of the hydrofoil, $x_{1}$ is its horizontal direction, and $x_{2}$ is its vertical direction. The computation domain is a rectangle area of the long side $7.5 \mathrm{~L}$ and the short side $2.8 \mathrm{~L}$. The distances of the leading edge to the short side and the long side are $1.5 L$ and $1.4 L$, respectively. The free stream velocity $U_{0}$ is constant.

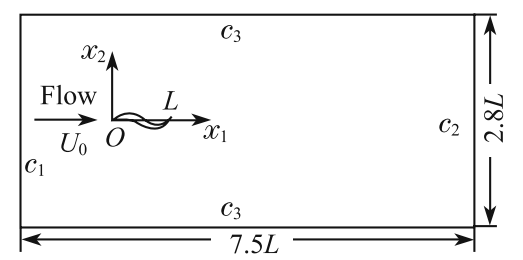

Fig. 1 Shape of hydrofoil NACA 65-010 and the initial location in computation domain

According to Akimoto and Miyata ${ }^{[22]}$, the wiggling motion is expressed by a progressive wave. The displacement $x_{\mathrm{b}}$ of the hydrofoil camber at the location $\left(x_{1}, 0\right)$ is prescribed by

$$
x_{\mathrm{b}}=A\left[-\left(\frac{x_{1}}{L}-1\right)^{2}+1\right] \cos \frac{2 \pi}{\lambda}\left(x_{1}-c t\right),
$$

where $x_{1}$ is the downstream distance from the leading edge of the hydrofoil, $A$ is the amplitude for the rear edge of the hydrofoil, $\lambda$ is the wavelength of the progressive wave, and $c$ is the phase 
velocity. The period $T$ of the wiggling motion is determined by $\lambda$ and $c$. The dimensionless period is $T^{*}=(\lambda / L) /\left(c / U_{0}\right)$.

\subsection{Governing equations}

The Navier-Stokes equations for a two-dimensional incompressible flow are written as

$$
\begin{aligned}
& \frac{\partial \boldsymbol{u}}{\partial t}+\boldsymbol{u} \cdot \nabla \boldsymbol{u}=-\nabla p+\frac{1}{R e} \nabla^{2} \boldsymbol{u}+\boldsymbol{f} \\
& \nabla \cdot \boldsymbol{u}=0
\end{aligned}
$$

where $\boldsymbol{u}$ is the velocity, $p$ is the pressure, and $\boldsymbol{f}$ is the external force. The Reynolds number is defined as $R e=L U_{0} / \nu$ with $\nu$ being the viscosity. The boundary conditions are given as follows. At the inlet boundary $c_{1}$, a uniform flow with the velocity $U_{0}$ is imposed, and the normal gradient of the pressure is set to be zero. At the outlet boundary $c_{2}$, the velocities are extrapolated from the interior, and the normal gradient of the pressure is also zero. At the lateral boundaries $c_{3}$, the gradient of the tangential velocity is zero, the normal velocity is zero, and the normal gradient of the pressure is also zero. A non-slip boundary condition is applied on the surface of the hydrofoil where the fluid velocity is the same as that of the foil.

\subsection{Numerical simulation}

We use the direct forcing method ${ }^{[19-21]}$ to simulate the flow fields of the wiggling hydrofoils. The central idea of the direct forcing method is to represent the effect of immersed bodies on flows by a virtual volume force $\boldsymbol{f}$ in Eq. (2). The governing equations are discretized in time as follows:

$$
\frac{\boldsymbol{u}\left(\boldsymbol{x}, t^{n+1}\right)-\boldsymbol{u}\left(\boldsymbol{x}, t^{n}\right)}{\Delta t}=\operatorname{rhs}\left(\boldsymbol{x}, t^{n+\frac{1}{2}}\right)+\boldsymbol{f}\left(\boldsymbol{x}, t^{n+1}\right),
$$

where $\operatorname{rhs}(\boldsymbol{x}, t)$ represents the sum of the convection term, the viscous term, and the pressure gradient at the intermediate time $t^{n+\frac{1}{2}}$ between $t^{n}$ and $t^{n+1}$. In the direct forcing method, two sets of grids are used. One is a Cartesian grid, on which the governing equations are solved. The other is a Lagrangian grid, which represents the geometry of the immersed bodies. The numerical procedure in the direct forcing method can be summarized as follows:

(i) Compute the explicit velocity estimation $\boldsymbol{u}$ without volume forces,

$$
\frac{\tilde{\boldsymbol{u}}-\boldsymbol{u}^{n}}{\Delta t}=-\frac{3}{2} \nabla_{h}(\boldsymbol{u} \boldsymbol{u})^{n}+\frac{1}{2} \nabla_{h}(\boldsymbol{u} \boldsymbol{u})^{n-1}-\nabla_{h} p^{n}+\frac{1}{2 R e} \nabla_{h}^{2}\left(\boldsymbol{u}^{n}+\tilde{\boldsymbol{u}}\right) .
$$

(ii) Calculate the volume forces at the Lagrangian points and spread them to the Eulerian points,

$$
\begin{aligned}
\frac{\boldsymbol{U}^{n+1}\left(\boldsymbol{X}_{k}\right)-\widetilde{\boldsymbol{U}}\left(\boldsymbol{X}_{k}\right)}{\Delta t} & =\sum_{j=1}^{M}\left(\sum_{x} \delta_{h}\left(\boldsymbol{x}-\boldsymbol{X}_{j}\right) \delta_{h}\left(\boldsymbol{x}-\boldsymbol{X}_{k}\right) \Delta s h^{2}\right) \boldsymbol{F}^{*}\left(\boldsymbol{X}_{j}\right) \\
\boldsymbol{f}^{*}(\boldsymbol{x}) & =\sum_{k=1}^{M} \boldsymbol{F}^{*}\left(\boldsymbol{X}_{k}\right) \delta_{h}\left(\boldsymbol{x}-\boldsymbol{X}_{k}\right) \Delta s .
\end{aligned}
$$

(iii) Correct the intermediate velocity using the volume forces and perform the pressure correction on the Cartesian grids,

$$
\begin{gathered}
\frac{\boldsymbol{u}^{*}-\tilde{\boldsymbol{u}}}{\Delta t}=\boldsymbol{f}^{*}, \\
\frac{\boldsymbol{u}^{* *}-\boldsymbol{u}^{*}}{\Delta t}=\nabla_{h} p^{n},
\end{gathered}
$$




$$
\begin{aligned}
\nabla_{h}^{2} p^{n+1} & =\frac{\nabla_{h} \cdot \boldsymbol{u}^{* *}}{\Delta t}, \\
\frac{\boldsymbol{u}^{n+1}-\boldsymbol{u}^{* *}}{\Delta t} & =-\nabla_{h} p^{n+1} .
\end{aligned}
$$

Here, the lowercase and uppercase letters are used to represent the variables defined on the Cartesian grids and the Lagrangian markers, respectively. In sum, the project method is used to decouple the solution of velocity and pressure. In time advancing, the Adams-Bashford and the Crank-Nicholson schemes are used for the convective and the diffusive terms, respectively. The spacial discretization is based on the finite volume approach.

A code for the direct forcing method has been developed and used to simulate the flows passing cylinders at low Reynolds numbers. The results obtained are in good agreement with the previous results ${ }^{[23-25]}$ as shown in Table 1.

Table 1 Comparison of the lift and drag coefficients in flow past a cylinder

\begin{tabular}{lccccc}
\hline \multirow{2}{*}{ Results } & \multicolumn{2}{c}{$R e=100$} & & \multicolumn{2}{c}{$R e=200$} \\
\cline { 2 - 3 } \cline { 5 - 6 } & $C_{\mathrm{d}}^{\text {avg }}$ & $C_{1}^{\text {max }}$ & & $C_{\mathrm{d}}^{\text {avg }}$ & $C_{1}^{\text {max }}$ \\
\hline Immersed boundary method (present) $_{\text {Lai and Peskin }}^{[23]}$ & 1.40 & 0.36 & & 1.38 & 0.70 \\
Pan and Damodaran $^{[24]}$ & 1.44 & 0.33 & & 0.63 \\
Henderson $^{[25]}$ & & & 1.37 & \\
\hline
\end{tabular}

To study the propulsive performance of the wiggling hydrofoil, we calculate the thrust coefficient, the lift coefficient, and the power coefficient. They are defined as

$$
\begin{aligned}
C_{\mathrm{t}} & =-\int \frac{F_{1} d s}{\rho U_{0}^{2} L / 2}, \\
C_{\mathrm{l}} & =\int \frac{F_{2} d s}{\rho U_{0}^{2} L / 2}, \\
C_{\mathrm{w}} & =-\int \frac{F_{2}\left(\frac{\partial x_{\mathrm{b}}}{\partial t}\right) d s}{\rho U_{0}^{3} L / 2},
\end{aligned}
$$

where $F_{1}$ and $F_{2}$ are the forces acting on the hydrofoil surface in the $x_{1}$ and $x_{2}$ directions, respectively. The propulsive efficiency is defined as

$$
\eta=\bar{C}_{\mathrm{t}} / \bar{C}_{\mathrm{w}},
$$

where $\bar{C}_{\mathrm{t}}$ and $\bar{C}_{\mathrm{w}}$ are the time-averaged values of the thrust coefficient and power coefficient.

\section{Numerical results}

The parameters in the numerical simulations are taken as follows. The free-stream velocity $U_{0}$ is equal to 1 , the dimensionless amplitude $A / L$ is 0.1 , the dimensionless wavelengths $\lambda / L$ are taken as 1 and 2 , and the phase velocity equals 8 . The typical configurations of the wiggling hydrofoil in one period for two different wavelengths are shown in Figs. 2(a) and 2(b), where the leading edges are fixed. It is observed that the amplitude of the wiggling hydrofoil increases from the leading edge to the rear edge, and a smaller wavelength represents a larger flexibility or curvature.

\subsection{Propulsive performance}

We first compare the thrust coefficient $C_{\mathrm{t}}$, the lift coefficient $C_{\mathrm{l}}$, and the power coefficient $C_{\mathrm{w}}$ for $R e=100$ with three different wave-numbers $\lambda=0.5,1,2$. The coefficients vary as functions of time, and they have constant amplitudes and frequencies, respectively, as shown in Figs. $3-5$. 


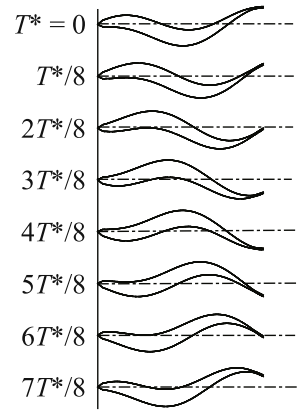

(a) $\lambda=1$

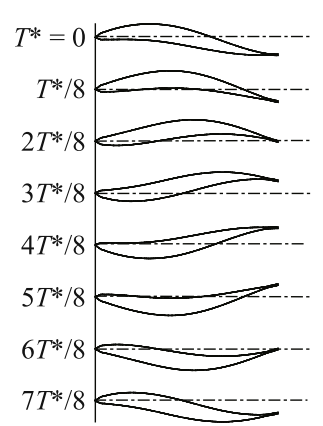

(b) $\lambda=2$

Fig. 2 Time variation of the wiggling hydrofoil

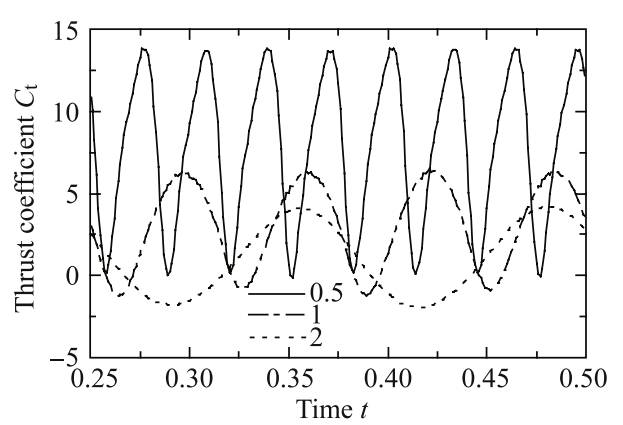

Fig. 3 Time variation of thrust coefficient

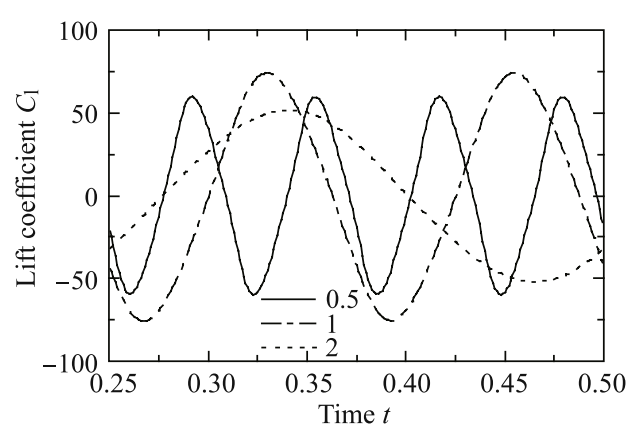

Fig. 4 Time variation of lift coefficient

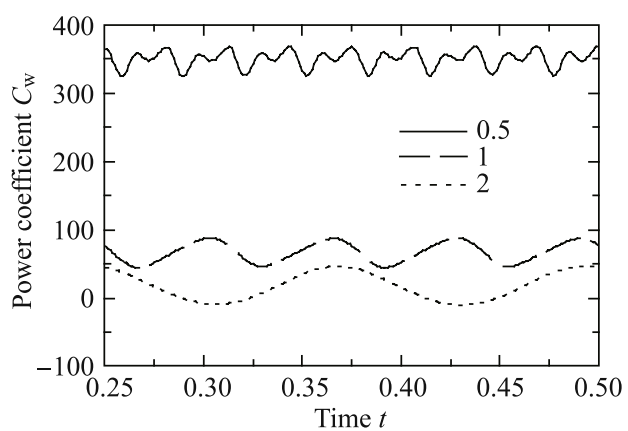

Fig. 5 Time variation of power coefficient

Figure 3 indicates that both the amplitudes and the frequencies of the thrust coefficients increase with the decrease of the wavelength. This implies that the flexibility could increase the propulsive force. Figure 4 shows that the amplitudes of the lift coefficients do not change much but the frequencies increase with the decrease of wavelength. Figure 5 indicates that the power coefficient at $\lambda=0.5$ is much larger than the ones at $\lambda=1,2$. Figure 5 also shows that the more flexible wiggling hydrofoil will consume more power.

To further understand the effects of the flexibility on the performance of the wiggling motion, we calculate the average thrust coefficient, the average power coefficient, and the propulsive efficiency for a larger range of wavelengths. Figures 6-8 plot the three parameters versus the wavelengths. It is found that both the average thrust coefficients and the average power coefficients decrease with the increase of the wavelength. They drop down fast at the initial stage, and then decrease slowly. However, the propulsive efficiency rises up slowly in the initial stage, and then decreases fast. It reaches the peak value of the propulsive efficiency at $\lambda=2$. This implies that the flexibility could enhance the thrust but high flexibility could suppress the propulsive efficiency. 
The average thrust coefficients, the average power coefficients, and the propulsive efficiencies are plotted against the Reynolds numbers for two different wavelengths $\lambda=1,2$ in Figs. 9-11, respectively. The average thrust coefficients reduce gradually with decreasing $R e$ at $20<R e$ $<200$, but they decrease at $R e<20$. The average power coefficients decrease remarkably at $R e<20$. The propulsive efficiencies become larger with the increasing Reynolds numbers.

In Figs. $9-11$, it is clearly observed that the average thrust coefficient at $\lambda=1$ is larger than that at $\lambda=2$, but the average power coefficient at $\lambda=2$ is less than that at $\lambda=1$. The propulsive efficiency at $\lambda=1$ is large at the initial stage and then it is less than that at $\lambda=2$. Therefore, the less flexible wiggling motion is more efficient in the larger-Reynolds-number flows.

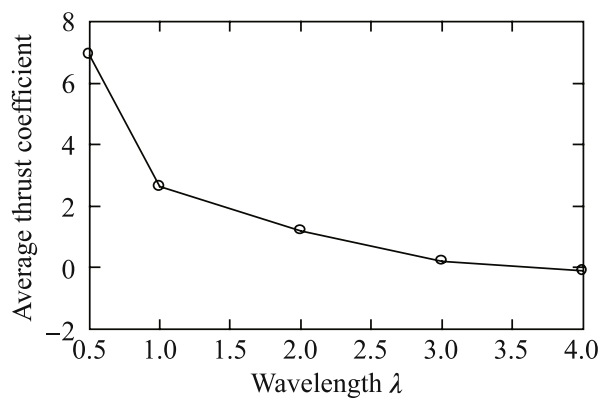

Fig. 6 Average thrust coefficient versus the progressive wavelength $(R e=100)$

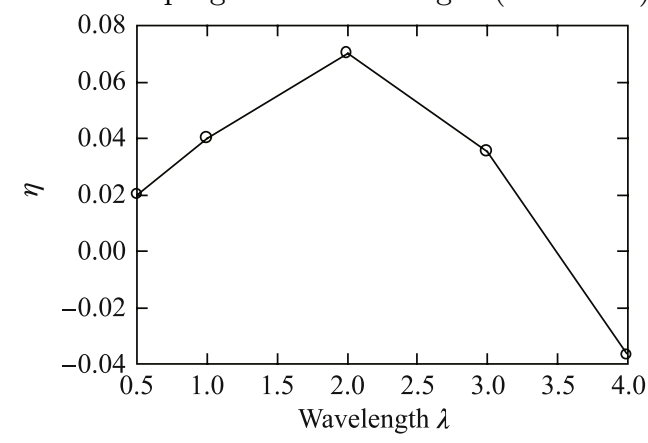

Fig. 8 Efficiency versus the progressive wavelength $(R e=100)$

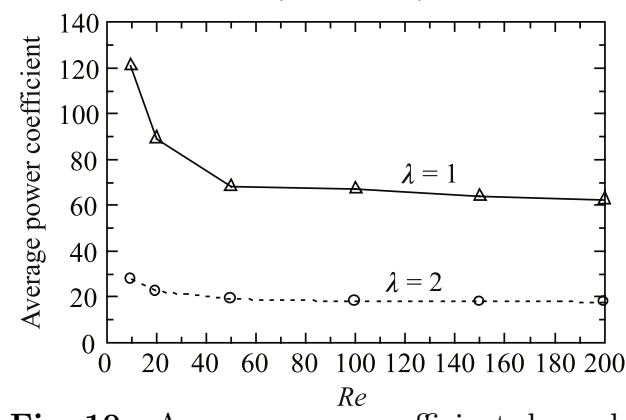

Fig. 10 Average power coefficient depending on $R e$ for $\lambda$

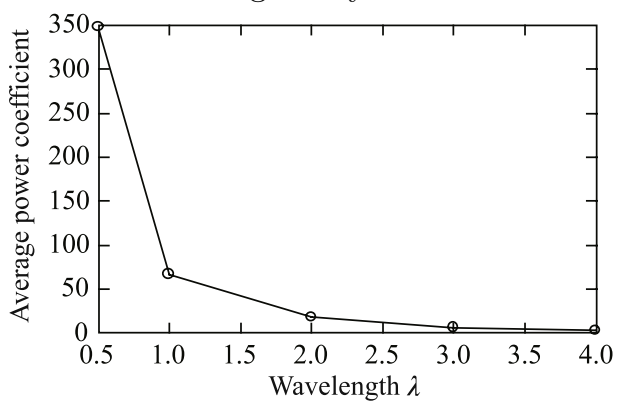

Fig. 7 Average power coefficient versus the progressive wavelength $(R e=100)$

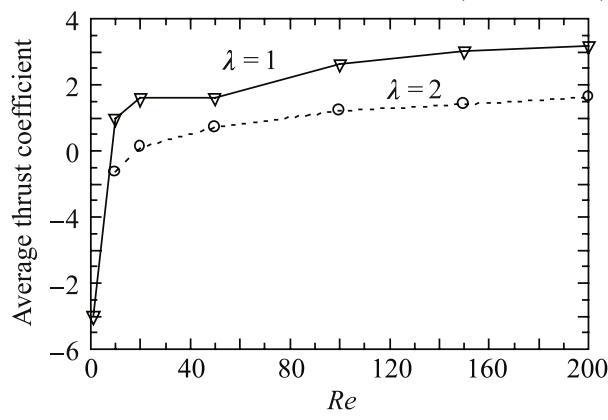

Fig. 9 Average thrust coefficient depending on $R e$ for $\lambda$

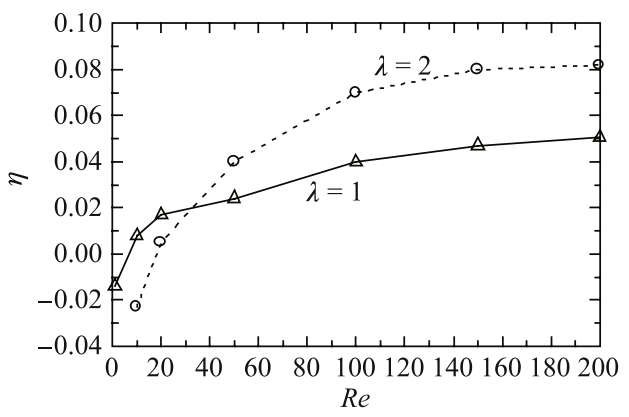

Fig. 11 Efficiency depending on $\operatorname{Re}$ for $\lambda$

\subsection{Flow patterns}

The flow patterns are plotted for the lower Reynolds numbers at $\lambda=0.5,1,2$, 4. At the lower Reynolds numbers, the viscous effects are expected to make the flow patterns different from the previous inviscid theories. The wavelengths ranging from $\lambda=0.5$ to $\lambda=4$ represent different flexibilities, which have different effects on the vortex patterns. The same contour level is taken for each plot to facilitate the comparison of different vortex structures. 
Figure 12 plots the vorticity isolines at $R e=100$ for the wavelengths $\lambda=0.5,1.0,2.0,4.0$. The vortices shed from the tails and form a wake downstream. For the smallest wavelength $\lambda=0.5$, the wake displays two rows of single vortices at the staggered arrangement. The rotation of the vortices is counter-clockwise in the top row, and the rotation of the vortices is clockwise in the bottom row. They are the well-known reversed Karman vortex streets. For the wavelength $\lambda=1.0$, the vortices on each row are paired without dislocation. The center line of the two rows is deflected from the horizontal line. They form the vortex dipoles. In term of Fig. 7, the thrust generated by the vortex diploes is smaller than that generated by the reversed Karman vortex streets. For the larger wavelengths $\lambda=2.0,4$, the vortices in the wakes are too weak to identify whether they are the reversed Karman vortex streets or the vortex dipoles. All of the wakes are of a jet-type and the vortices become weaker as the vortices move to downstream.

Figure 13 plots the vorticity isolines at the wavelength $\lambda=2$ for the Reynolds numbers $R e$ $=10,50,100,150$. The vorticities in the wake become weaker at the smaller Reynolds numbers than the ones at the larger Reynolds numbers due to the viscous diffusion. For $R e=10$, the vorticities are diffused so much that neither the reversed Karman vortex street nor the vortex dipole could be observed. For $R e=150$, the vortices are less diffused, and thus two rows of single vortices are observed evidently. Therefore, the Reynolds numbers have an important influence on the vortex streets.

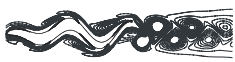

(a) $\lambda=0.5$

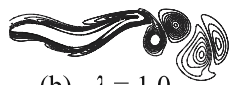

(b) $\lambda=1.0$

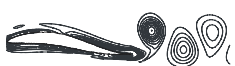

(c) $\lambda=2.0$

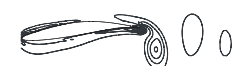

(d) $\lambda=4.0$

Fig. 12 Vorticity isolines at $\lambda=0.5,1.0,2.0,4.0$ (where the vorticity value ranges from -100 to 100 with intervals of 10 dotted and solid lines denote negative and positive contours)

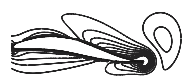

(a) $R e=10$

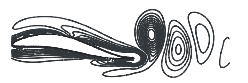

(b) $R e=50$

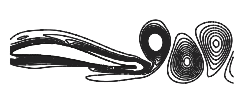

(c) $R e=100$

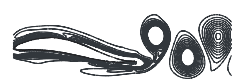

(d) $R e=150$

Fig. 13 Vorticity isolines at $R e=10,50,100,150$ (where the vorticity value ranges from -50 to 50 with intervals of 5 dotted and solid lines denote negative and positive contours)

\section{Conclusions}

An immersed boundary method is used to simulate the flows around a wiggling hydrofoil NACA 65-010. The wiggle motion is described by a progressive wave, where the wavelength represents the flexibility of the wiggle motion, and the amplitude represents the magnitude of the wiggle motion. It is found that the thrust generated by the wiggle motion is dependent on the wavelength from the numerical results. The thrust coefficient becomes larger with the smaller wavelengths, and the power coefficient exhibits the same trend as the thrust coefficient. However, the propulsive efficiency does not exhibit such monotonicity, and it takes a maximum value at a certain wavelength, where the viscosity plays an important role. Two distinguishing flow patterns are observed in the wakes. The reversed Karman vortex streets are associated with the larger thrust, and the vortex dipoles are associated with the smaller thrust.

\section{References}

[1] Lighthill, M. J. Note on the swimming of slender fish. Journal of Fluid Mechanics 9(2), 305-317 (1960)

[2] Lighthill, M. J. Hydromechanics of aquatic animal propulsion. Annual Review of Fluid Mechanics 1, 413-466 (1969)

[3] Freyumth, P. Propulsive vortical signature of plunging and pitching airfoils. AIAA Journal 26(7), 881-883 (1988) 
[4] Jones, K. D., Dohring, C. M., and Platzer, M. F. Experimental and computational investigation of the Knoller-Betz effect. AIAA Journal 36(7), 1240-1246 (1998)

[5] Jones, K. D., Castro, B. M., Mahmoud, O., Pollard, S. J., Platzer, M. F., Neef, M. F., Gonet, K., and Hummel, D. A collaborative numerical and experimental investigation of flapping-wing propulsion. AIAA Paper 0706 40th Aerospace Sciences Meeting \& Exhibit, Reno, Nevada (2002)

[6] Lighthill, M. J. Large-amplitude elongated-body theory of fish locomotion. Proc. R. Soc. Lond. B 179, 125-138 (1971)

[7] Wu, T. Y. Hydrodynamics of swimming propulsion. Journal of Fluid Mechanics 46(2), 521-544 (1971)

[8] Newman, J. N. The force on a slender fish-like body. Journal of Fluid Mechanics 58(4), 689-702 (1973)

[9] Cheng, J. Y., Zhuang, L. X., and Tong, B. G. Analysis of swimming 3-D waving plate. Journal of Fluid Mechanics 232(11), 341-355 (1991)

[10] Trianafyllou, G. S., Trianafyllou, M. S., and Grosenbaugh, M. A. Optimal thrust development in oscillating foils with application to fish propulsion. Journal of Fluids and Structure 7(2), 205-224 (1993)

[11] Trianafyllou, M. S. and Trianafyllou, G. S. An efficient swimming machine. Science American 272(3), 1-6 (1995)

[12] Yang, Y. H., Yin, Z. X., and Lu, X. Y. Flow visualization over a 2-D traveling wave wall (in Chinese). Journal of Experiments in Fluid Mechanics 19(2), 84-90 (2005)

[13] Dong, G. J. and Lu, X. Y. Numerical analysis on the propulsive performance and vortex shedding of fish-like traveling wavy plate. International Journal for Numerical Methods in Fluids 48(12), 351-373 (2005)

[14] Tong, B. G., Sun, M., and Yin, Z. X. A brief review on domestic research developments in bio-fluid dynamics of animal flying and swimming (in Chinese). Chinese Journal of Nature 27(4), 191-198 (2005)

[15] Wang, L., Wang, M., and Wu, C. J. Numerical simulation of self-propelled oscillating foil (in Chinese). Acta Mechanica Solida Sinica 27(SI), 110-114 (2006)

[16] Li, L. and Yin, Z. X. Experiments on propulsive characteristics of the caudal-fin models of carangiform fish in cruise (in Chinese). Journal of Experiments in Fluid Mechanics 22(1), 1-5 (2008)

[17] Uchiyama, T. and Kikuyama, K. Numerical simulation for the propulsive performance of a submerged wiggling micromachine. Journal of Micromechanics and Microengineering 14(11), 1537$1543(2004)$

[18] Mittal, R. and Iaccarino, G. Immersed boundary methods. Annual Review of Fluid Mechanics 37, 239-261 (2005)

[19] Kim, J., Kim, D., and Choi, H. An immersed-boundary finite-volume method for simulations of flow in complex geometries. Journal of Computational Physics 171(1), 132-150 (2001)

[20] Su, S. W., Lai, M. C., and Lin, C. A. An immersed boundary technique for simulating complex flows with rigid boundary. Computers and Fluids 36(2), 313-324 (2007)

[21] Uhlmann, M. An immersed boundary method with direct forcing for the simulation of particular flows. Journal of Computational Physics 209(2), 448-476 (2005)

[22] Akimoto, H. and Miyata, H. Finite-volume simulation of a flow about a moving body with deformation. Proceeding 5th International Symposium on Computational Fluid Dynamics, Sendai, Japan, 1, 13-18 (1993)

[23] Lai, M. C. and Peskin, C. S. An immersed boundary method with formal second order accuracy and reduced numerical viscosity. Journal of Computational Physics 160(2), 705-719 (2000)

[24] Pan, H. and Damodaran, M. Parallel computation of viscous incompressible flows using Godunovprojection method on overlapping grids. International Journal for Numerical Methods in Fluids 39(5), 441-463 (2002)

[25] Henderson, R. D. Details of the drag curve near the onset of vortex shedding. Physics of Fluids 7(9), 2102-2104 (1995) 